\title{
The Effect of Clean and Microbiota-Free Environment on Host Immune Response
}

\author{
Cassie C. He ${ }^{1}$ and Joshua S. Yuan ${ }^{2 *}$ \\ ${ }^{1}$ A\&M Consolidated High School, College Station, TX, USA \\ ${ }^{2}$ Texas A\&M Agrilife Synthetic and Systems Biology Innovation Hub, Texas A\&M University, College Station, TX, USA
}

*Corresponding author: Joshua S. Yuan, Texas A\&M Agrilife Synthetic and Systems Biology Innovation Hub, Texas A\&M University, College Station, TX 77843, USA; E-mail: syuan@tamu.edu

\begin{abstract}
Accompanied with improved living conditions and hygiene practices, there is a steady and steep increase of occurrence of allergic diseases in developed countries. Hygiene Hypothesis, which states that lack of microbes at the early childhood attributes the improper development of a healthy immune system in adulthood, has been formulated to explain this clinical observation. The mechanisms underlying Hygiene Hypothesis remain obscure largely due to the complex interface between environmental factors and genetic composition in clinical studies and animal model systems. Here, we explored the potential of deploying a simple plant system to examine the effect of clean environment on host immune system. We compared the growth phenotype and the immune response of the model plant Arabidopsis thaliana grown on autoclaved soil (clean environment) and non-autoclaved soil (dirty environment). We observed that plants grown on autoclaved soil exhibited a weaker immune response than plants grown on non-autoclaved soil at the adulthood stage. Plants grown on autoclaved soil showed severer infestation by fungal gnats, the common Arabidopsis pests, and less uniformity in size than those grown on non-autoclaved soil. These data suggest that individuals living in a clean environment may develop a dysfunctional immune system at the adulthood compared to those grown in a dirty environment. The autoclave process kills soil microbiota, which might be a reason for altered growth and immunity. Thus, our studies suggest that host immune response and microbiota should be considered when studying the underlying mechanisms of Hygiene Hypothesis.
\end{abstract}

Received date: February 16, 2017

Accepted date: April 29, 2017

Published date: May 2, 2017

Citation: Yuan, J.S., et al. The Effect of Clean and Microbiota-Free Environment on Host Immune Response. (2017) J Environ Health Sci 3(2): 1- 5.

DOI: $10.15436 / 2378-6841.17 .1370$

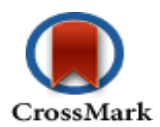

Keywords: Hygiene hypothesis; Immune response; Microbiota; Clean environment; Plants

\section{Introduction}

Compared to developing countries, Western developed countries appear to have a steady increase of allergic diseases in recent years (Strachan, 1989). Hygiene Hypothesis was formulated as a possible explanation to the phenomenon. The hypothesis states that living an ultraclean lifestyle without harmless microbes may cause children to be prone for development of allergic diseases (Strachan, 1989). This also provides a basis of using benign infectious organisms to treat or prevent diseases involving immune dysregulation, autoimmunity or chronic inflammation (Liu and Leung, 2006; Schaub et al., 2006; Vercelli, 2006). Hygiene Hypothesis suggests that exposure to microbes early in life reduces the risks of developing allergic diseases by boosting the activity of the healthy immune system activ- ity. When children are developing their immune system, they don't experience the harmless microbes that they need to. Thus, when children grow older, their body reacts in ways that they shouldn't, result in high risks of development of allergies. Studies have shown that people living in developing countries with more exposure to microbes are less likely to have allergic diseases (Riedler et al., 2001). Recently, the American Academy of Allergy, Asthma and Immunology surveyed urban and rural families. They found that families that lived in high income parts of cities and clean environments had higher rates of allergies. However, families that lived on rural farms without modern cleaning technologies were reported to develop substantially lower allergies than their urban counterparts (Bloomfield et al., 2006). However, the mechanisms underlying Hygiene Hypothe- 
sis remain obscure largely due to the complex interface between environmental factors and genetic composition in clinical studies and animal model systems.

Several animal models have been developed to define the molecular basis of Hygiene Hypothesis. Unfortunately, the results are often confusing because no two models are identical in terms of genetic background, growth conditions, and time and dose of exposure to environmental signals (Vercelli, 2006). One study suggested that mice that lived in antiseptic labs (cleaning environment) were less healthy than those living in sewers, farms, and other outdoor areas (less hygienic environment). The wild-caught rats were associated with increased IL-4 production and attenuated proliferative and pro-inflammatory responses of splenocytes (Lesher et al., 2006). However, when comparing the immune system of laboratory animals and wild animals, the principal concern is that wild animals are (i) genetically different from and (ii) more genetically diverse than their inbred laboratory cousins.

A simplified and cost-effective model system in which extrinsic and intrinsic variations can be readily and tightly controlled may provide a novel avenue to validate and understand the molecular basis underlying Hygiene Hypothesis. Arabidopsis thaliana, a mustard weed found by roadsides and in disturbed land, has been developed as a laboratory model system to study diverse arrays of biological questions (Jones et al., 2008). $A$. thaliana possesses many advantages, including the genetic tractability, complete genome sequence, whole-genome Gene Chips, and the collection of whole-genome knockout lines with very low cost. Notably, the homogenous Arabidopsis population as a result of self-fertile lifestyle, enables repeated phenotyping of the identical genotypes under diverse controlled environmental conditions (Pang and Meyerowitz, 1987). Importantly, despite the obviously different lifestyles, plants and animals bear commonalities in fundamental biochemical and cellular activities. For example, plants have developed a highly similar perception and immune system with animals in response to pathogen attacks (Ausubel, 2005). This feature makes it extremely well suited for studying environmental effects, a determining factor of direct importance to Hygiene Hypothesis.

Here we explored the potential of deploying A.thaliana to study the effect of clean environment on host immune responses and determine whether it could providing insights for understanding Hygiene Hypothesis, a fundamental yet clinically challenging question in a cost efficient way. We have used the Arabidopsis transgenic plants expressing an immune responsive marker gene fused with a firefly luciferase reporter ( $\mathrm{Li}$ et al., 2014). When these plants are challenged by microbes, the on-set of plant defense or immunity will activate the immune marker gene, leading to expression of luciferase reporter and production of bioluminescence, which could be readily captured by a charge-coupled device (CCD) camera or quantified by a luminometer. We compared the immune response, by monitoring the microbe-induced luminescence, of these plants grown in either clean or dirty environment. The low maintenance cost, easy set of the experiments and homogenous feature of plant population make it possible to minimize the complication of genetic and environmental variations that are often difficult to control in clinical studies and animal models. The knowledge gained from this research could provide an evolutionary framework on the role of immunity and microbiota on Hygiene Hypothesis, and generate insight for the development of novel preventive and therapeutic strategies for medical treatment of allergies and autoimmune diseases.

\section{Materials and Methods}

\section{Plant materials and growth}

Arabidopsis thaliana transgenic plants expressing a firefly luciferase reporter gene under the control of the FRK1 promoter (FRK1::LUC) were used (Li et al., 2014). The FRK1 (flagellin-induced receptor-like kinase 1) gene can be quickly activated by microbes and serve as a marker gene to indicate the immune response.

The plant seeds were surface-sterilized using $5 \%$ bleach for $10 \mathrm{~min}$ and put in pots containing soil (Metro Mix 360) with about 16 plants per pot. One set of soil and water $\left(\mathrm{ddH}_{2} \mathrm{O}\right)$ were autoclaved at $121^{\circ} \mathrm{C}$ and $15 \mathrm{psi}$ for 20 minutes to kill germs as the sterile growth condition. One set of FRK1::LUC transgenic plant seeds were germinated on the sterilized soil and one set on the non-sterilized soil. The plants on the sterilized soil were watered with autoclaved water whereas those on non-sterilized soil were watered with non-autoclaved water (Figure 1A). The plants were grown in a growth chamber with condition of $23^{\circ} \mathrm{C}$, $60 \%$ relative humidity and $75 \mu \mathrm{E} \mathrm{m}^{-2} \mathrm{~s}^{-1}$ light with a 12-hr light and 12-hr dark photoperiod for four weeks.

\section{Microbial growth and treatment to activate immune re- sponse}

We used bacterial Pseudomonas syringae pv. tomato DC3000 hrcC and bacterial flagellin peptide flg22 as a trigger to active immune response in FRK1::LUC transgenic plants. The $h r c C$ strain is a nonpathogenic plant bacterium, which induces plant immune response and activates FRK $1:: L U C$ activity. Flg22 is a synthesized peptide corresponding to a part of bacterial flagellin, which also induces immune response and activates the FRK $1:$ LUC activity (Li et al., 2014).

The $h r c C$ bacterium was first grown on a petri dish containing the King's B (KB) medium with rifampicin $(50 \mu \mathrm{g}$ $\left.\mathrm{ml}^{-1}\right)$ at $28^{\circ} \mathrm{C}$ for 48 hours. Then a single colony was inoculated into $4 \mathrm{ml}$ of liquid $\mathrm{KB}$ medium with rifampicin $\left(50 \mu \mathrm{g} \mathrm{ml}^{-1}\right)$ on a roller drumat $28^{\circ} \mathrm{C}$ for 36 hours. Bacteria were pelleted by centrifugation at $24^{\circ} \mathrm{C}, 4,000 \mathrm{rpm}$ for 10 minutes, washed with 2 $\mathrm{ml}$ of $\mathrm{ddH}_{2} \mathrm{O}$ for twice, and diluted to $\mathrm{OD}_{600}=0.5$ using $\mathrm{ddH}_{2} \mathrm{O}$. The leaves of four-week-old plants were hand-infiltrated using a needleless-syringe with $\mathrm{ddH}_{2} \mathrm{O}$ (control), $200 \mathrm{nM} \mathrm{flg} 22$, or $h r c C$ at the $\mathrm{OD}_{600}=0.5$.

\section{Luciferase measurement}

Leaves were cut at 8 hours and 24 hours after inoculation to measure luciferase activity. The individual leaves were transferred to each well of a 96-well plate and sprayed with 0.2 $\mathrm{mM}$ luciferin, the substrate of luciferase. The plate was put in the dark for 20 minutes to quench the autoflurorescence, and the bioluminescence signal was read by a luminometer (Perkin Elmer, 2030 Multilabel Reader, Victor X3).

\section{Data analysis}

At least ten leaves for each time point of each treatment were subjected to luciferase measurement. The lowest and highest data were discarded as the potential outliers. The data from 
the left eight leaves were used to calculate the mean \pm standard deviation (SD) with Microsoft Excel software and the significance was calculated with Student's t-test (http://www.physics. csbsju.edu/stats/t-test.html).

\section{Results}

As outlined in figure 1, we have proposed to monitor the immune response using Arabidopsis FRK1::LUC transgenic plants grown on autoclaved soil (clean environment) and non-autoclaved soil (dirty environment). We hypothesized that plants grown from clean and dirty environment may show different sensitivity to immune response elicitation. There are several potential advantages in this system to study Hygiene Hypothesis. First, the seeds used in this assay were harvested from a single self-pollinated plant. Thus, the individual plants used for comparison of their immune response are genetically identical. Second, plants were grown side-by-side in a strictly controlled environment with fixed humidity, light and temperature. Thus, this greatly reduced the variations from environmental factors. Third, measurement of luciferase activity from the FRK1::LUC transgenic plants is a straight forward experiment and can be readily carried out and quantified. Thus, this provides a reliable and easy assay to monitor the immune response. Finally, the germ-free and clean environment verses dirty environment is easily set up by autoclaving the soil and water. Taken together, although life styles in plants and humans differ in large, the system used in this study could provide insights into underlying mechanisms of Hygiene Hypothesis. Figure 1

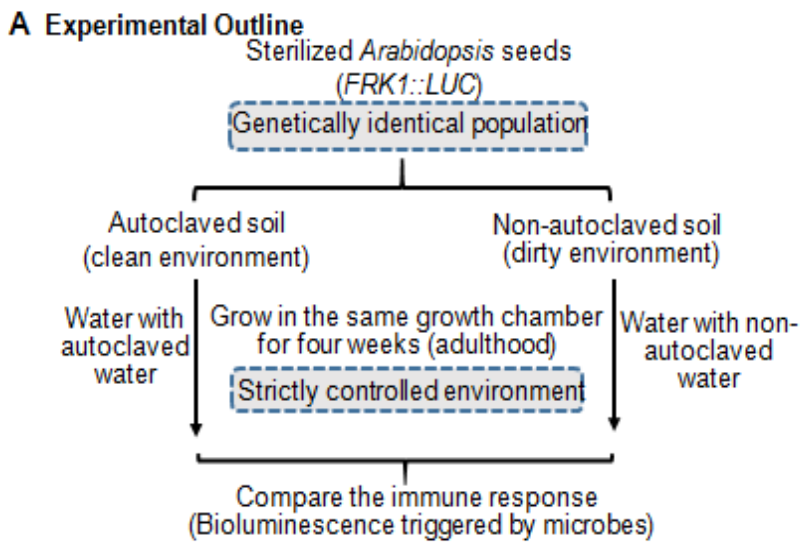

B Hypothesis: Autoclaved soil is a germ-free environment. Plants grown in the autoclaved soil resemble people living in a clean environment. Non-autoclaved soil has lots of germs. Plants grown in non-autoclaved soil resemble people living in a dirty environment.

Materials: Arabidopsis plants carrying the immune marker gene FRK1 fused with the firefly luciferase gene (FRK1::LUC) were used as hosts. The plant bacteria Pseudomonas syringae pv. tomato DC3000 hrcC or bacterial flagellin peptide flg22 was used to induce plant immune response. A luminometer machine was used to detect bioluminescence production of FRK1: LUC plants.

Experiments: Grow plants in autoclaved and non-autoclaved soil for four weeks; infect plants with bacterial hrcC or flg22 peptide; compare the immune response of FRK1::LUC plant by a luminometer machine; and finally perform data analysis. The experiments were repeated three times.

Figure 1. (A) Scheme of the experiments. (B) Highlights of hypothesis, experiments and materials used in the experiments.
Flg22 is a peptide derived from bacterial flagellin and hrcC is a non-pathogenic bacterium, both of which serve as immune triggers on Arabidopsis. Among four independent experiments, we have consistently observed that plants grown on either non-autoclaved soil or autoclaved soil showed the enhanced luciferase activity after flg22 or $h r c C$ treatment compared to plants without inoculation or inoculated with $\mathrm{H}_{2} \mathrm{O}$ control (Figure 2). Thus, our results indicate that the luciferase activity in FRK1::LUC transgenic plants was derived from the elicitation of immune response by microbes but not from wounding by hand-inoculation process. Importantly, in three out of four experiments, we repetitively observed that plants grown on non-autoclaved soil exhibited a higher luciferase activity in response to flg22 or $h r c C$ treatment than plants grown on autoclaved soil. In a typical experiment in Figure 2, the non-autoclaved soil grown plants had a higher luciferase activity in response to flg22 at 12 hours after treatment than autoclaved soil grown plants (Figure 2A). At 24 hours after treatment, the non-autoclaved soil grown plants had a higher luciferase activity in response to both flg22 and $h r c C$ (Figure 2B). The data indicate that plants grown in the clean environment may have reduced immune reactions in response to pathogen infections compared to plants grown in the clean environment. Notably, we observed that plants grown on autoclaved soil most times had slightly higher $F R K:: L U C$ activity than plants grown on non-autoclaved soil in the absence of any treatment (First columns in Figure 2A and 2B). Figure 2
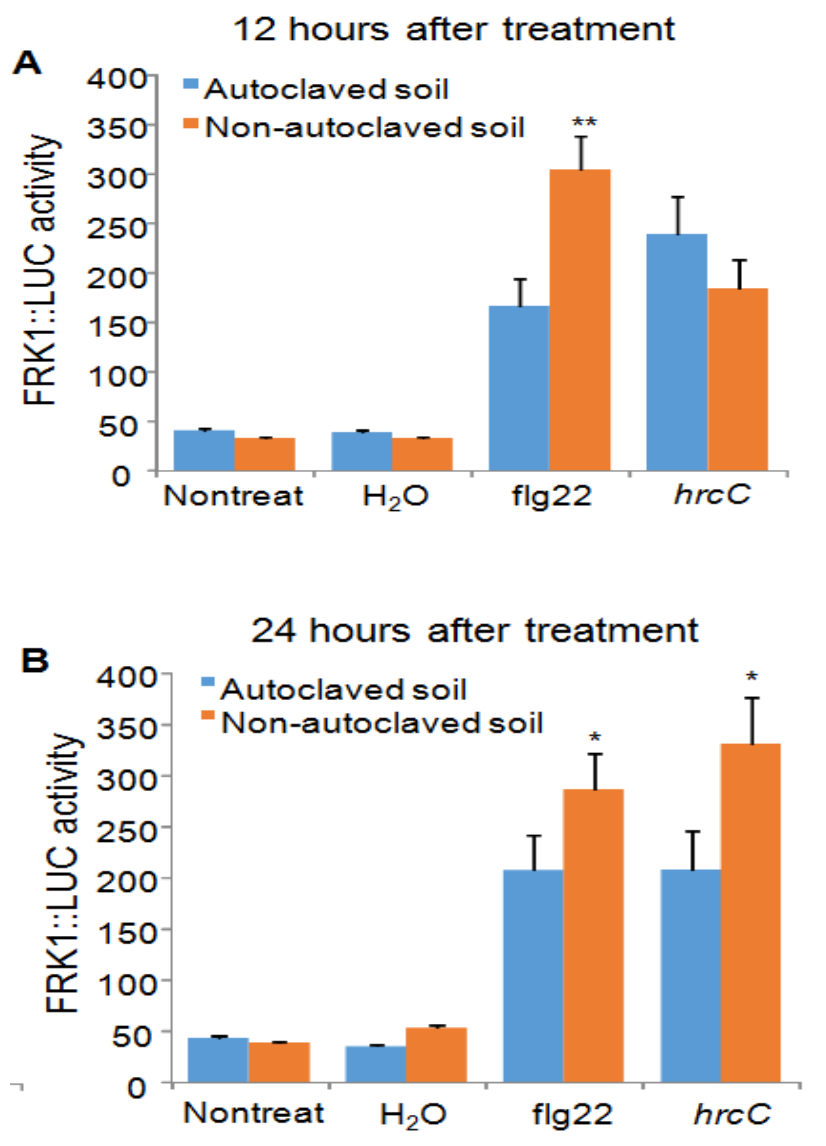

Figure 2. Reduced immune response in plants grown on autoclaved soil compared to those grown on non-autoclaved soil. Leaves of four-weekold FRK1::LUC plants grown on different soil were hand-infiltrated with $\mathrm{ddH}_{2} \mathrm{O}, 200 \mathrm{nM}$ flg 22 or $h r c C$ at $\mathrm{OD}_{600}=0.5$ using a needleless syringe. Non-treated leaves were included as controls. Leaves were collected at 12 (A) or 24 (B) hours after treatment. The data are shown as mean \pm SD with Student's t-test from 8 leaves for each treatment. * 
indicates $\mathrm{p}<0.05$ and $* *$ indicates $\mathrm{p}<0.01$ when compared to plants on autoclaved soil. The experiments were repeated independently four times. The experimental data from three (II, III and IV repeats) out of four repeats showed similar trend. The data from the first repeat was not reliable because of bad inoculation.

Fungus gnats (Bradysia spp.) are one of commonly seen pests of Arabidopsis in greenhouses (Bush et al., 2006). The larval stage fungus gnats appear as little worms and feed on tender roots, fungi and organic matter and decay tissues in soil. Fungus gnat larvae chew on leaves that come in contact with soil surface. A notable symptom from fungus gnat infestation is the visible holes on leaves contacting soil. Adult fungus gnats seen as small flies do not harm the plants but lay eggs, which soon hatch into more larvae. In our experimental setting, we consistently observed that plants grown on autoclaved soil had more severe infections from fungus gnats than plants grown on non-autoclaved soil (Figure 3A \& 3B). Among 30 plants grown on autoclaved soil, 14 plants (46.7\%) were bitten by fungus gnats (Figure. $3 \mathrm{C}$ ). However, none of plants grown on non-autoclaved soil were damaged by fungus gnats. This is consistent with the above observation that plants from autoclaved soil possess weak immune response, which may make it more susceptible to fungus gnat infestation. Notably, we used the healthy and undamaged plants for immune response presented in Figure 2. In addition, we observed that plants grown on autoclaved soil were not very uniform in size, in particular when plants were before four-week-old stage (Figure 3A). Some plants are relatively big, where as some are relatively small. In contrast, plants grown on non-autoclaved soil were relatively uniform (Figure 3A). The size of plants is not related to fungus gnat infestation since some plants bitten by fungus gnats grew relatively big and vice versa. Figure 3

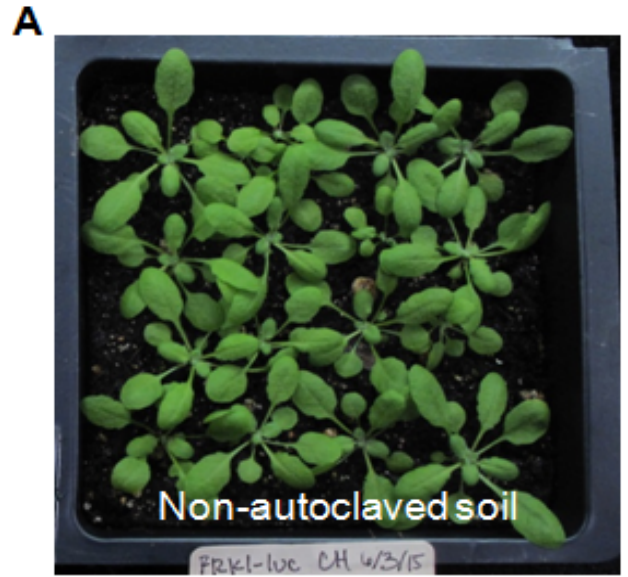

B

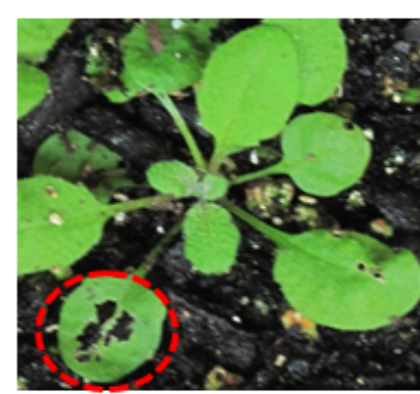

C Total plants Damaged plants Percentage (\%)

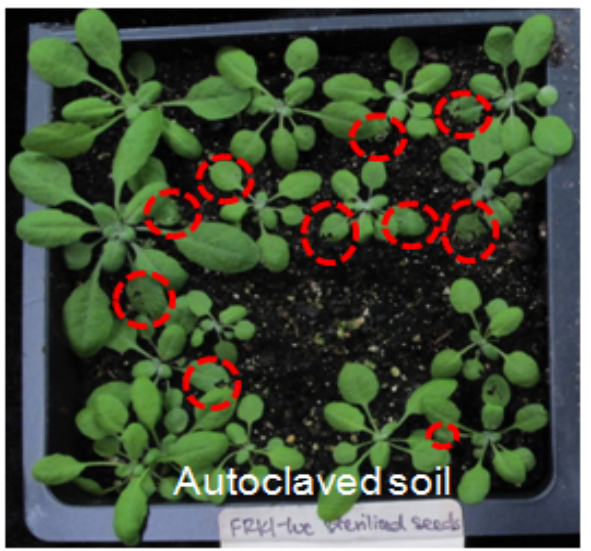

Autoclaved Nonautoclaved

30

31

14

0

46.7

0

Figure 3. Plants grown on autoclaved soil are much more sensitive to fungus gnat infestations compared to those grown on non-autoclaved soil. (A) The view of plants in pots with non-autoclaved or autoclaved soil. The damage caused by fungus gnatsare indicated with red dotted circles in the image. Notably, plants grown on non-autoclaved soil are more uniform than plants on autoclaved soil. (B )A close-up view of leaves bitten by fungus gnats. (C) Percentage of plants infested by fungus gnats.

\section{Discussion and Conclusion}

From our experiments, we have observed

1. Plants grown on autoclaved soil (clean environment) exhibited a weakened immune response upon infection compared to those grown on non-autoclaved soil (dirty environment).

2. Plants grown on autoclaved soil (clean environment) showed severe infestations by fungus gnats, the common Arabidopsis pests in greenhouse, compared to those grown on non-autoclaved soil (dirty environment).

3. Plants grown on autoclaved soil (clean environment) are not uniform in size compared to those grown on non-autoclaved soil (dirty environment).
These data suggest that individuals grown up in a clean environment may develop a dysfunctional immune system at the adulthood so that they respond to microbes or pathogens differently from those grown up in a dirty environment. We also observed that plants grown in a clean environment have more variation in size than plants in a dirty environment. It is possible that the high temperature and pressure during autoclave process may disrupt organic components in soil. The plants grown on autoclaved soil may lack sufficient nutrients to live on. Apparently, the autoclave process kills soil microbiota, which has been suggested to play an important role in supporting the health and 
growth for plants and animals ( $\mathrm{Wu}$ and $\mathrm{Wu}, 2012)$. The altered soil microbiota may also impact the host immune system and render plants easier to get infected by fungal gnats. The autoclave and non-autoclaved soil introduces additional factors to be considered in order to study the effect of clean environment on host immune response. The future experiment that we propose to do is to grow plants in non-autoclaved soil, treat plants with or without microbes at seedling stage (childhood), and then compare the immune response between treated and non treated plants at their adult stage.

Human allergic reactions are often caused by immune intolerance. For example, asthma is caused by patient's immune system reacted to the environmental exposure such as pollen too "strongly". Interestingly, plants grown on clean environment had slightly higher immune response than plants grown on dirty environment in the absence of infections (First columns in Figure $2 \mathrm{~A}$ and $2 \mathrm{~B}$ ). This indicates that plants in the clean environment may already have certain immune reactions to the nonpathogenic stimuli, which may resemble human allergic reactions. Nevertheless, host immune response and microbiota should be considered when studying the underlying mechanisms of Hygiene Hypothesis.

Acknowledgment: We thank Drs. Paul deFigueiredo and Koichi Kobayashi in the College of Medicine at Texas A\&M University for the discussion of Hygiene Hypothesis, Dr. Wenwei Lin for the technical assistance, and anonymous reviewers for the comments to improve the manuscript

\section{Reference}

1. Ausubel, F.M. Are innate immune signaling pathways in plants and animals conserved? (2005) Nat Immunol 6(10): 973-979.

PubMed | CrossRef | Others

2. Bloomfield, S.F., Stanwell-Smith, R., Crevel, R.W., et al. Too clean, or not too clean: the hygiene hypothesis and home hygiene. (2006) Clin Exp Allergy 36(4): 402-425.

PubMed | CrossRef $\mid$ Others

3. Bush, J., Jander, G., Ausubel, F.M. Prevention and control of pests and diseases. (2006) Methods Mol Biol 323: 13-25.

PubMed | CrossRef $\mid$ Others

4. Jones, A.M., Chory, J., Dangl, J.L., et al. The impact of Arabidopsis on human health: diversifying our portfolio. (2008) Cell 133(6): 939943.

PubMed | CrossRef | Others

5. Lesher, A., Li, B., Whitt, P., et al. Increased IL-4 production and attenuated proliferative and pro-inflammatory responses of splenocytes from wild-caught rats (Rattus norvegicus). (2006) Immunol Cell Biol 84(4): 374-382.

PubMed | CrossRef | Others

6. Li, F., Cheng, C., Cui, F., et al. Modulation of RNA Polymerase II Phosphorylation Downstream of Pathogen Perception Orchestrates Plant Immunity. (2014) Cell Host Microbe 16(6):748-758.

PubMed | CrossRef | Others

7. Liu, A.H., Leung, D.Y. Renaissance of the hygiene hypothesis. (2006) J Allergy Clin Immunol 117(5): 1063-1066.

PubMed | CrossRef | Others

8. Pang, P.P., Meyerowitz, E.M. Arabidopsis Thaliana - a Model System for Plant Molecular-Biology. (1987) Bio-Technol 5: 1177-1181.

PubMed | CrossRef $\mid$ Others

9. Riedler, J., Braun-Fahrlander, C., Eder, W., et al. Exposure to farming in early life and development of asthma and allergy: a cross-sectional survey. (2001) Lancet 358(9288): 1129-1133.

PubMed | CrossRef | Others

10. Schaub, B., Lauener, R., von Mutius, E. The many faces of the hygiene hypothesis. (2006) J Allergy Clin Immunol 117(5): 969-977. PubMed | CrossRef | Others

11. Strachan, D.P. Hay fever, hygiene, and household size. (1989) BMJ 299(6710): 1259-1260.

PubMed | CrossRef | Others

12. Vercelli, D. Mechanisms of the hygiene hypothesis--molecular and otherwise. (2006) Curr Opin Immunol 18(6): 733-737.

PubMed | CrossRef | Others

13. Wu, H.J., Wu, E. The role of gut microbiota in immune homeostasis and autoimmunity. (2012) Gut Microbes 3(1): 4-14.

PubMed | CrossRef | Others
Ommega Online Publishers

Journal Title: Journal of Environment and Health Science (JEHS)

Journal Short Name: J Environ Health Sci
Journal ISSN: 2378-6841

E-mail: environmentalscience@ommegaonline.org

Website: www.ommegaonline.org 